\title{
Metabolic Alterations Associated With Antipsychotic Use-A Descriptive Study and Comparison between Haloperidol and Olanzapine in Schizophrenic and Bipolar Patients
}

\author{
Sérgio Luiz Prior ${ }^{1}$, Antonio Ricardo de Toledo Gagliardi ${ }^{1}$, Marcos Montani Caseiro ${ }^{1}$ and Pedro Luis Prior ${ }^{2 *}$ \\ ${ }^{1}$ Centro Universitário Lusiadas, Department of Psychiatry, Santos/SP, Brazil \\ ${ }^{2}$ Universidade Federal de São Paulo, Department of Medicine, São Paulo/SP, Brazil
}

\begin{abstract}
Introduction: Antipsychotic drugs are essential to psychiatric health in many conditions, such as schizophrenia and bipolar disorder. However, they present several side effects, such as motor dystonia and "Parkinson-like" behavior (especially in first generation antipsychotics) and metabolic alterations leading to increased body weight, type 2 diabetes and dyslipidemias. Second generation antipsychotics are widely regarded as more damaging in metabolic side effects, though there are few studies comparing if first generation antipsychotics can provoke similar changes in metabolism.
\end{abstract}

Methods: An observational-transversal-descriptive study was conducted in a total of 63 patients in use of their first generation (haloperidol- $\mathrm{N}=27$ ) or second generation antipsychotic-olanzapine- $\mathrm{N}=36$ ). Blood samples were collected to assess the following parameters-Fasting Glucose, HDL Cholesterol, LDL Cholesterol, Triglycerides and Basal Insulin. Anthropometric measurements of abdominal and neck circumference, as well as weight were also taken and compared between both groups.

Statistical analyses: If variables were considered of normal distribution, the Student $t$-test and variance analyses (ANOVA) were performed to test for significant differences between groups. If samples were considered non-parametric, U Mann-Whitney test, Kruskal-Wallis test, Qui-square for extensive table, or Fish's test were utilized. Statistical significance was considered $5 \%$ on all analyzes $(p<0.05)$.

Results: There were no statistically significant differences between both groups using either first and second generation antipsychotics., in relation to anthropometric measurements (abdominal circumference measurements $p=0.56$, U Mann-Whitney test), metabolic status (HOMA index $p=0.12$, HDL cholesterol $p=0.27$, basal glycemia $p=0.08$, BMI $p=0.51$, triglycerides $p=0.12$, Chi-square for extensive tables).

Discussion: Metabolic alterations occurred in both groups, which support the literature findings which show a high prevalence of metabolic alteration in antipsychotic medication users.

Keywords: Antipsychotic drugs; Schizophrenia; Bipolar disorder; Metabolic syndrome

\section{Introduction}

Schizophrenia and Bipolar Disorder are two psychiatric conditions of great interest in modern times, not only because of their prevalence in psychiatric practice, but because, if untreated by any means, both are associated with higher physical and mental comorbitites, loss in productivity and quality of life and even increase risk of death [1-7].

Bipolar disorder is defined by alterations between episodes depression and mania/hypomania. Depression is characterized by predominant sadness, loss of will and action, weight changes, sleep changes and suicidal ideation. Mania and hypomania are characterized by sudden expansion of affect, mood elevation, inflated self-esteem, decreased need for sleep, increased speed of thought, and psychomotor agitation [1,5]. The exact prevalence is still unknown (considered $2.1 \%$ by the World Health Organization). Bipolar disorder normally presents itself in early adulthood, and is correlated with physical and social risks, increasing risk of death by external causes, and suicide by $25 \%$ when compared with general population [8].

Schizophrenia is a psychiatric illness defined by changes in course of thought and sense perception, normally presenting itself with delusions and sensory hallucinations. Prevalence is $1 \%$ worldwide, generally presenting itself in early adulthood-between 20-30 years of age [2].

\section{Pharmacological treatments and risk}

Treatment for bipolar disorder and schizophrenia may include dopamine receptor antagonists. First appearing in the 1950's and 60's, the first generation of dopamine antagonists included chlorpromazine and haloperidol. Both medications were useful for managing agitation, delusional thoughts, and hallucinatory symptoms in psychiatric patients. Side effects included extrapyramidal motor symptoms including tremors and dyskinesia $[9,10]$. They were also correlated with change in metabolic status, with weight gain and hypercholesterolemia [5-7].

A second generation of dopamine antagonists was introduced in the 1970's. They offered alternatives for control of both hallucinatory and behavioral symptoms of schizophrenia, and with fewer propensities

${ }^{*}$ Corresponding author: Pedro Luis Prior, Universidade Federal de São Paulo, Department of Medicine, R. Armando Sales de Oliveira, number 32 - Santos/SP, Brazil ZIP Code - 11050070, E-mail: pedrolsprior@gmail.com

Received July 03, 2014; Accepted July 30, 2014; Published August 06, 2014

Citation: Prior SL, Gagliardi ART, Caseiro MM, Prior PL (2014) Metabolic Alterations Associated With Antipsychotic Use-A Descriptive Study and Comparison between Haloperidol and Olanzapine in Schizophrenic and Bipolar Patients. J Pharmacovigilance 2: 143. doi:10.4172/2329-6887.1000143

Copyright: ( 2014 Prior SL, et al. This is an open-access article distributed under the terms of the Creative Commons Attribution License, which permits unrestricted use, distribution, and reproduction in any medium, provided the original author and source are credited. 
to induce motor side-effects [10]. However, they have been associated greater incidence of metabolic changes such as hypercholesterolemia, hypertriglyceridemia, increased incidence of type 2 diabetes, hypertension and increased general myocardial infarction risk. As documented by many studies, these metabolic alterations collaborate for increased risk of mortality by cardiovascular causes, as well as increased risk of morbidity [5-7,9-11].

There have been few studies comparing the metabolic alterations caused by first and second generation antipsychotics, trying to determine if there is a greater incidence of comorbitites with use of either type of medication [12-15].

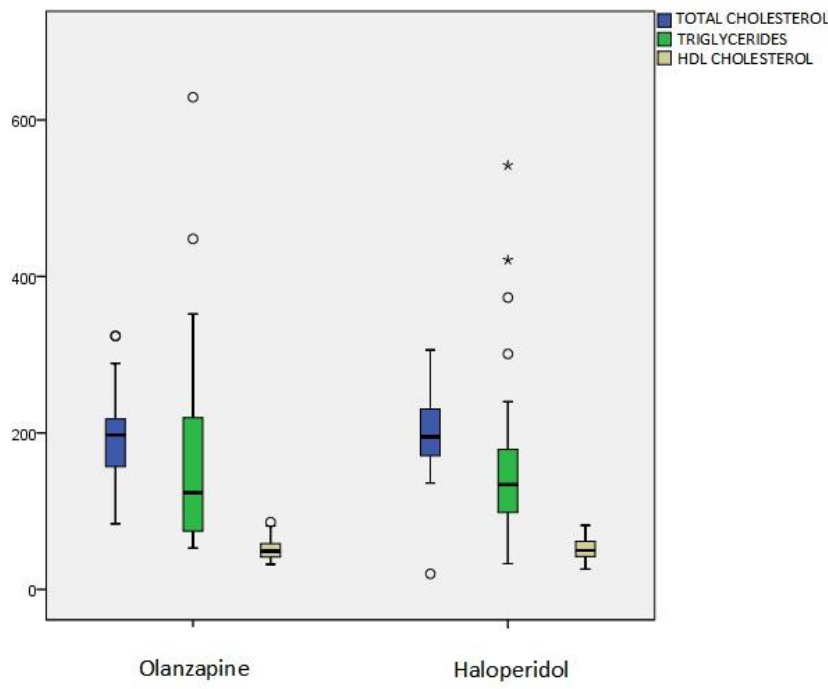

Figure 1: Distribution of medium total cholesterol, HDL cholesterol amongst antipsychotic users, according to antipsychotic.

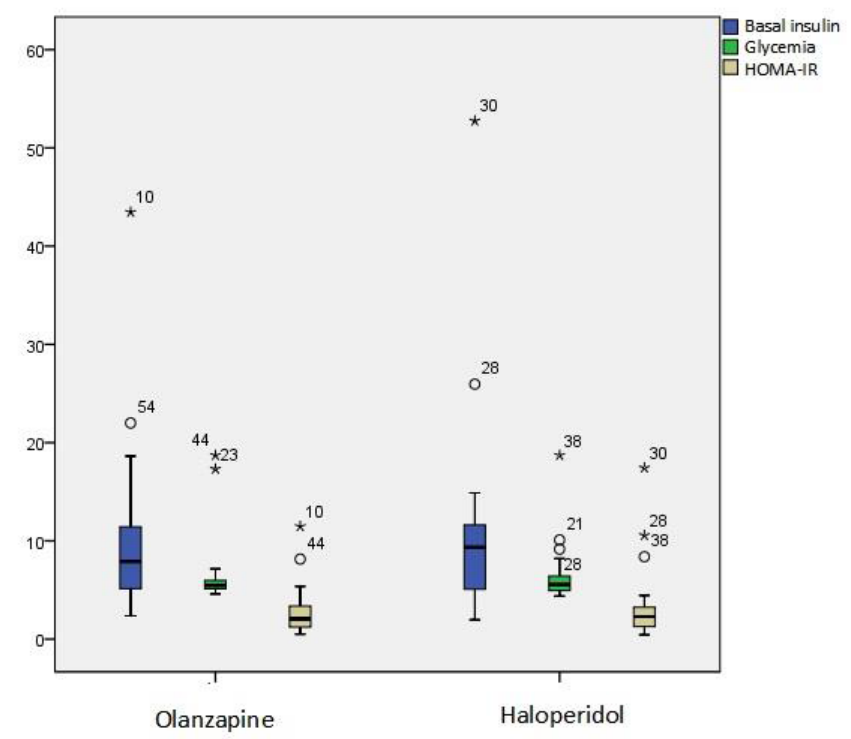

Figure 2: Correlation between medium Basal Insulin Glycemia and HOMAIR amongst antipsychotic users, according to antipsychotic.

\section{Objective}

To determine and compare metabolic status and alterations between two groups of patients: one in current use of first generation antipsychotics and the other second generation antipsychotics in chronic psychiatric treatment for schizophrenia or bipolar disorder.

\section{Methods}

The study was observational-transversal-descriptive, with a total of 63 enrolled patients $(\mathrm{N}=63)$, with psychiatric diagnosis of either bipolar disorder or schizophrenia, in use of either typical or atypical antipsychotics drugs for at least 5 years, and enrolled in a municipal service in Santos, São Paulo, specialized in providing consultations and medication-Seção Núcleo de Apoio Psicossocial II. The patients were asked to participate, and gave written consent via form, authorizing blood collection and result divulgence. The study and the consent form were both approved by the Ethics and Human Research Committee of the Santos Prefecture, as well as he Ethics Committee of Centro Universitário Lusíadas.

Exclusion criteria were any psychiatric comorbitites other than those mentioned in inclusion criteria, and current use of any medication to treat diabetes or any form of dyslipidemia. History of metabolic alterations and diseases before onset of psychiatric symptoms (diabetes or hypertriglyceridemia) were also considered exclusion criteria. One single blood collection was performed after patients consented, in which the following parameters were analyzed: fasting glucose, HDL cholesterol, LDL cholesterol, basal insulin and triglycerides in serum concentrations (Figure 1).

The National Cholesterol Education Program Adult Treatment Panel III (NCEP-ATP-III) criteria were used, and metabolic parameters were considered altered in the following criteria: 1) HDL cholesterol lower than $40 \mathrm{mg} / \mathrm{dl}$ for males and lower than $50 \mathrm{mg} / \mathrm{dl}$ for females, 2) Triglycerides higher than $150 \mathrm{mg} / \mathrm{dl}$, 3) Fasting glucose higher than $110 \mathrm{mg} / \mathrm{dl}$. LDL cholesterol was considered high if over $130 \mathrm{mg} / \mathrm{dl}$ [16].

Basal insulin and fasting glucose were used for calculation of HOMA (Homeostatic Model Assessment) index and Homa Beta Percentage (HOMA B-\%), used for measuring type 2 diabetes incidence risk in individual persons (Figure 2). HOMA indexes higher than 1 show increased insulin resistance, and correlated with higher risk for developing type 2 diabetes [17].

Anthropometrics measurements were taken as well, and patients were classified according to WHO BMI Indexes (weight kilograms divided by height in centimeters square), in which patients are classified in straits: normal weight if BMI between 18.5 and 25, overweight if BMI between 25 and 30, Obesity type I if BMI between 30 and 35, Obesity type II if BMI between 35 and 40 and Obesity for BMI above 40 [18]. The risk for cardiovascular disease is increased directly with increased BMI [19]. Measurements of abdominal circumference were utilized as the main index pointing to greater metabolic risk, and ATP III-NCEP criteria were used as parameters- $102 \mathrm{~cm}$ for males and $88 \mathrm{~cm}$ for females [16] (Figure 3).

Statistical analyses were performed using Kolmogorov-Smirnov (with Lillifors correction) and Shapiro-Wilk tests to test for normal distribution of variables. If variables were considered of normal distribution, the $t$ Student test and variance analyses (ANOVA) were performed to test for significant differences between groups. If samples were considered non-parametric, Mann-Whitney U test, KruskalWallis test, Qui-square for extensive table, or Fish's test were utilized. Statistical significance was considered $5 \%$ on all analyzes $(p<0.05)$. 


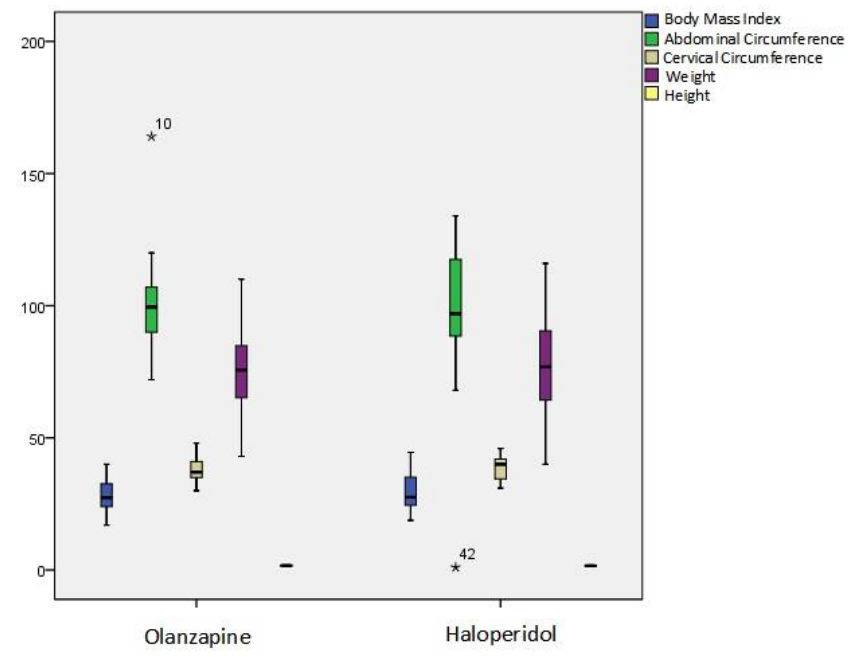

Figure 3: Distribution of medium body mass index, abdominal and cervical circumferences, height and weight amongst antipsychotic users, according to antipsychotic.

\begin{tabular}{|c|c|c|}
\hline Characteristics & n & $\%$ \\
\hline Gender & & \\
\hline Male & 19 & 30.2 \\
\hline Female & 44 & 69.8 \\
\hline Age (years) & & \\
\hline$<40$ & 17 & 27 \\
\hline $40-60$ & 38 & 60.3 \\
\hline$>60$ & 8 & 12.7 \\
\hline & & \\
\hline Body Mass Index (BMI) & & \\
\hline Eutrofic & 20 & 31.7 \\
\hline Overweight & 15 & 23.8 \\
\hline Obesity I & 16 & 25.4 \\
\hline Obesity II & 8 & 12.7 \\
\hline Obesity III & 4 & 6.3 \\
\hline & & \\
\hline Diagnosis & & \\
\hline Paranoid schizophrenia (F20) & 40 & 63.5 \\
\hline Bipolar affective disorder (F31) & 23 & 36.5 \\
\hline
\end{tabular}

Table 1: Sociodemographic characteristics - gender, age, body mass index and diagnosis of the studied population as a whole $(\mathrm{N}=63)$.

\section{Results}

Of the 63 patients in total, $\mathrm{N}=36$ (53.9\%) made use of the atypical antipsychotic olanzapine at maximum dose $20 \mathrm{mg}$ daily, and $\mathrm{N}=27$ (46.1\%) made use of the typical antipsychotic haloperidol at daily dose of $20 \mathrm{mg}$ daily. There were no statistically significant differences between groups in the following sociodemographic parameters: age, gender, time of use of antipsychotic, smoking or alcohol use in quantity or frequency or significant family history of cardiovascular disease. Other clinical standards were not statistically different between both groups. Olanzapine group: Time of untreated psychosis $-4.23 \pm 6.4$ years, time of antipsychotic use $-12.1 \pm 5$ years. Haloperidol group: Time of untreated psychosis $-5.4 \pm 4.4$ years, time of antipsychotic use $-10.1 \pm 3$ years. Sociodemographic data can be seen in table 1 .
There were, however, no statistical differences between typical and atypical groups in any of the analyzed criteria, as seen in tables 2 and 3 , in relation to anthropometric measurements (abdominal circumference measurements $\mathrm{p}=0.56$, Mann-Whitney $\mathrm{U}$ test), metabolic status (HOMA index $\mathrm{p}=0.12$, HDL cholesterol $\mathrm{p}=0.27$, basal glycemia $\mathrm{p}=0.08$ , BMI $p=0.51$, triglycerides $p=0.12$, Chi-square for extensive tables).

\section{Discussion}

The data obtained from this study shows that both groups present metabolic alterations consistent with hypercholesterolemia, hypertriglyceridemia, type 2 diabetes and obesity, however with no significant difference between typical and atypical medication users.

There are limitations to our study, as it does not take into consideration the effect of other psychoactive substances used by bipolar patients (valproate acid, which was the case in our sample) in lipid profile. There is also the possibility that other environmental factors may have played an important role on changing lipid profile: such as antidepressants and mood stabilizers of chronic use.

Second generation antipsychotics present an alternative treatment, as they allow control of behavioral symptoms and less motor side effects when compared with first generation antipsychotics. This leads to better patient adherence and greater improvement in patient outcome [9].

Weight gain and greater cardiovascular risk in antipsychotics -prescribed psychiatric patients, whether it be first or second generation, are shown in many studies as important contributors to higher risk of mortality in this population [4-6].

The goal of modern psychiatry is to improve patient outcome and quality of life. However, results such as shown here demonstrate need to implement new actions of modern health care: the mental health

\begin{tabular}{|c|c|c|}
\hline & \multicolumn{2}{|c|}{ Diagnostic-n (\%) } \\
\hline & Schizophrenia (F20) & Bipolar disorder (F32) \\
\hline \multicolumn{3}{|l|}{ Gender } \\
\hline Male & $15(78.9)$ & $4(21.1)$ \\
\hline Female & $25(56.8)$ & $19(43.2)$ \\
\hline \multicolumn{3}{|l|}{ Age (years) } \\
\hline Less than 40 & $13(76.5)$ & $4(23.5)$ \\
\hline More than 40 & $27(58.7)$ & $19(41.3)$ \\
\hline \multicolumn{3}{|l|}{ IMC } \\
\hline Eutrofic & $10(50)$ & $10(50)$ \\
\hline Overweight/Obese I-II-III & $30(69.8)$ & $13(30.2)$ \\
\hline \multicolumn{3}{|l|}{ HOMA-IR } \\
\hline Normal & $22(55)$ & $18(45)$ \\
\hline Hyperinsulinemia & $18(78.3)$ & $5(21.7)$ \\
\hline \multicolumn{3}{|l|}{ HOMA-Beta } \\
\hline Normal & $32(59.3)$ & $22(40.7)$ \\
\hline Hyperinsulinemia & 8 (88.9) & $1(11.1)$ \\
\hline \multicolumn{3}{|l|}{ Glycemia } \\
\hline Normal & $22(61.1)$ & $14(38.9)$ \\
\hline Resistent & $18(66.7)$ & $9(33.3)$ \\
\hline \multicolumn{3}{|l|}{ LDL Cholesterol } \\
\hline Normal & $19(54.3)$ & $16(45.7)$ \\
\hline Elevated & $21(75)$ & $7(25)$ \\
\hline \multicolumn{3}{|l|}{ Triglycerides } \\
\hline Normal & $26(63.4)$ & $15(36.6)$ \\
\hline Elevated & $14(63.5)$ & $8(36.5)$ \\
\hline
\end{tabular}

Table 2: Incidence of metabolic alterations according to psychiatric diagnosis $(\mathrm{N}=63)$. 
Citation: Prior SL, GagliardiART, Caseiro MM, Prior PL (2014) Metabolic Alterations Associated With Antipsychotic Use-ADescriptive Study and Comparison between Haloperidol and Olanzapine in Schizophrenic and Bipolar Patients. J Pharmacovigilance 2: 143. doi:10.4172/2329-6887.1000143

Page 4 of 4

\begin{tabular}{|c|c|c|}
\hline & \multicolumn{2}{|c|}{ Neuroleptic - $\mathbf{n}(\%)$} \\
\hline & Olanzapine & Haloperido \\
\hline \multicolumn{3}{|l|}{ Gender } \\
\hline Male & $10(52.6)$ & $9(47.4)$ \\
\hline Female & $26(59.1)$ & $18(40.9)$ \\
\hline \multicolumn{3}{|l|}{ Age (years) } \\
\hline Less than 40 & $12(70.6)$ & $5(29.4)$ \\
\hline More than 40 & $24(52.2)$ & $22(47.8)$ \\
\hline \multicolumn{3}{|l|}{ IMC } \\
\hline Eutrofic & $12(60)$ & $8(40)$ \\
\hline Overweight/Obese I-II-III & $24(55.8)$ & $19(44.2)$ \\
\hline \multicolumn{3}{|l|}{ HOMA-IR } \\
\hline Normal & $24(60)$ & $16(40)$ \\
\hline Hyperinsulinemia & $12(52.2)$ & $11(47.8)$ \\
\hline \multicolumn{3}{|l|}{ HOMA-Beta } \\
\hline Normal & $33(61.1)$ & $21(38.9)$ \\
\hline Hyperinsulinemia & $3(33.3)$ & $6(66.7)$ \\
\hline \multicolumn{3}{|l|}{ Glycemia } \\
\hline Normal & $22(61.1)$ & $14(38.9)$ \\
\hline Resistent & $14(51.9)$ & $13(48.1)$ \\
\hline \multicolumn{3}{|l|}{ LDL Cholesterol } \\
\hline Normal & $19(54.3)$ & $16(45.7)$ \\
\hline Elevated & $17(60.7)$ & $11(39.3)$ \\
\hline \multicolumn{3}{|l|}{ Triglycerides } \\
\hline Normal & $23(56.1)$ & $18(43.9)$ \\
\hline Elevated & $13(59.1)$ & $9(40.9)$ \\
\hline
\end{tabular}

5. Jupfer DJ (2005) The increase medical burden in bipolar disorder. JAMA 293: 2598-2530.

6. Fenn HH, Bauer MS, Alshuher L, Evans DR, Williford WO, et al. (2005) A medical comorbidity and health related quality of life in bipolar disorder across the adult age span. J Affect Disord 86: 47-60.

7. Casey DE (2005) Metabolic issues and cardiovascular disease in patients with psychiatric disorders. Am J Med 2:15S-22S.

8. Jamison KR (2000) Suicide and bipolar disorder. J Clin Psychiatry 9:47-51.

9. Cuerda C, Velasco C, Merchán-Naranjo J, García-Peris P, Arango C (2014) The effects of second-generation antipsychotics on food intake, resting energy expenditure and physical activity. Eur J Clin Nutr 68: 146-152.

10. Subotnik KL, Ventura J, Gretchen-Doorly D, Hellemann GS, Agee ER, et al (2014) The impact of second-generation antipsychotic adherence on positive and negative symptoms in recent-onset schizophrenia. Schizophr Res S0920 9964(14)00365-X.

11. Laursen TM, Munk-Olsen T, Nordentoft M, Mortensen PB (2007) Increased Mortality among patients admitted with major psychiatric disorders: a registerbased study comparing mortality in unipolar depressive disorder. bipolar affective disorder, schizoaffective disorder, and schizophrenia. J Clin Psychiatry 68: 899-907.

12. Said MA, Sulaiman AH, Habil MH, Das S, Bakar AK, et al. (2012) Metabolic syndrome and cardiovascular risk among patients with schizophrenia receiving antipsychotics in Malaysia. Singapore Med J 53: 801-807.

13. Said MA, Hatim A, Habil MH, Zafidah W, Haslina MY, et al. (2013) Metabolic syndrome and antipsychotic monotherapy treatment among schizophrenia patients in Malaysia. Prev Med 57 Suppl: S50-S53.

14. Cerit C, Vural M, Bos Gelmez SÜ, Ozten E, Aker AT, et al. (2010) Metabolic syndrome with different antipsychotics: a multicentre cross-sectional study. Psychopharmacol Bull 43: 22-36.

15. Bobes J, Arango C, Aranda P, Carmena R, Garcia-Garcia M, et al. (2012) Cardiovascular and metabolic risk in outpatients with schizoaffective disorder treated with antipsychotics: results from the CLAMORS study. Eur Psychiatry 27: $267-274$.

professional must not only administer proper medication and manage psychiatric symptoms, but also manage patient cardiovascular risk, whether it is by prescribing proper medication, encouraging eating habit changes and proper exercise routine.

\section{Acknowledgement}

Fundação de Amparo à Pesquisa do Estado de São Paulo (FAPESP), Associação Fundo de Incentivo à Pesquisa (AFIP), Conselho Nacional de Desenvolvimento e Tecnológico (CNPq).

\section{References}

1. Angst J Gamma A, Benazzi F, Ajdacic V, Eich D, et al. (2003) Toward a redefinition of subthreshold bipolarity: epidemiology and proposed criteria for bipolar-II, minor bipolar disorders and hypomania. See comment in PubMed Commons below J Affect Disord 73: 133-146.

2. Jablensky A (1997) The 100-year epidemiology of schizophrenia. See comment in PubMed Commons below Schizophr Res 28: 111-125.

3. Barrett RJ (1998) Conceptual foundations of schizophrenia: I. Degeneration. See comment in PubMed Commons below Aust N Z J Psychiatry 32: 617-626.

4. Hung $\mathrm{Cl}$, Liu CY, Hsiao MC, Yu NW, Chu CL (2014) Metabolic syndrome among psychiatric outpatients with mood and anxiety disorders. BMC Psychiatry 14:185.

16. (2001) Executive Summary of The Third Report of The National Cholestero Education Program (NCEP) Expert Panel on Detection, Evaluation, And Treatment of High Blood Cholesterol In Adults (Adult Treatment Panel III) JAMA 285: 2486-2497.

17. Smith RC, Lindenmayer JP, Davis JM, Kelly E, Viviano TF, et al. (2009) Effects of olanzapine and risperidone on glucose metabolism and insulin sensitivity in chronic schizophrenic patients with long-term antipsychotic treatment: a randomized 5-month study. J Clin Psychiatry 70: 1501-1513.

18. WHO (2006) BMI Classification: Global Database on Body Mass Index. World Health Organization.

19. Herrmann J, Gersh BJ, Goldfinger JZ, Witzenbichler B, Guagliumi G, et al (2014) Body mass index and acute and long-term outcomes after acute myocardial infarction (from the Harmonizing Outcomes With Revascularization and Stents in Acute Myocardial Infarction Trial). Am J Cardiol 114: 9-16. 